\title{
Explorative analysis of a predictive score for patients with metastatic, castration resistant prostate cancer undergoing radioligand therapy with [177Lu]Lu-labeled prostate-specific membrane antigen
}

Kai Huang ( $\square$ kai.huang@charite.de)

Charite Universitatsmedizin Berlin https://orcid.org/0000-0003-1743-8693

Imke Schatka

Charite Universitatsmedizin Berlin

Julian M.M. Rogasch

Charite Universitatsmedizin Berlin

Randall L. Lindquist

Charite Universitatsmedizin Berlin

Maria De Santis

Charite Universitatsmedizin Berlin

\section{Barbara Erber}

Charite Universitatsmedizin Berlin

Piotr Radojewski

Charite Universitatsmedizin Berlin

\section{Winfried Brenner}

Charite Universitatsmedizin Berlin

Holger Amthauer

Charite Universitatsmedizin Berlin

Original research

Keywords: predictive score, radioligand therapy, prostate cancer, [177Lu]Lutetium-PSMA

Posted Date: April 27th, 2020

DOI: https://doi.org/10.21203/rs.3.rs-24704/v1

License: (c) (i) This work is licensed under a Creative Commons Attribution 4.0 International License.

Read Full License 


\section{Abstract}

Background: Up to $60 \%$ of patients with metastatic, castration-resistant prostate cancer (mCRPC) treated with [177Lu]Lu-labeled prostate-specific membrane antigen (PSMA) radioligand therapy (RLT) achieve partial biochemical response with a decrease of $>50 \%$ in prostate-specific antigen (PSA) levels. The remaining fraction of patients, however, do not respond to RLT. The aim of this explorative analysis was to identify pretherapeutic factors for the prediction of response.

Methods: 46 patients (age $=68$ years [53-87]) with mCRPC who consecutively underwent RLT with [177Lu]Lu-PSMA (median applied activity $=6 \mathrm{GBq}$ [2.9-6.2]) were included and analysed retrospectively. Association of different clinical and laboratory factors and parameters from pretherapeutic 68GalliumPSMA positron emission tomography (PET) with the outcome of RLT was tested (Fisher's test). Outcome was defined as PSA changes 8 weeks after the second RLT (partial response [PR], PSA decrease $>50 \%$; progressive disease [PD], PSA increase $\geq 25 \%$; stable disease [SD], others). Significant risk factors were combined in a predictive score.

Results: Median pretherapeutic PSA was $79 \mathrm{ng} / \mathrm{ml}$. Thirty percent of the patients showed a posttreatment PR (median: 73\% PSA decrease), 35\% SD (median: 17\% PSA decrease) and 35\% PD (median: $42 \%$ PSA increase). Significant predictors for PD were alkaline phosphatase (ALP) $>135 \mathrm{U} / \mathrm{I}(p=0.030)$, $P S A>200 \mathrm{ng} / \mathrm{ml}(\mathrm{p}=0.036)$, and maximum standardized uptake value (SUVmax) of the "hottest lesion" in pretherapeutic PET $<45(\mathrm{p}=0.005)$. The predictive score including PSA, ALP, and SUVmax could separate 2 distinct groups of patients: $\leq 2$ risk factors ( $81 \%$ PR or SD, $19 \%$ PD) and 3 risk factors (10\% PR or SD, $90 \%$ PD).

Conclusion: The presented predictive score allowed a pretherapeutic estimate of the expected response to RLT. This is hypothesis generating. Prospective trials are needed to test these predictive risk factors

\section{Background}

Prostate cancer has the highest incidence and the third highest cancer related mortality for men in Europe [1]. In the last decade, several treatment options for patients with metastatic, castration-resistant prostate cancer (mCRPC) were established, including abiraterone, enzalutamide, docetaxel and cabazitaxel [2]. More recently, radioligand therapy (RLT) with $\left({ }^{177} \mathrm{Lu}\right)$ Lu-labeled prostate-specific membrane antigen (PSMA) was developed and has shown promising results with regards to prostate-specific antigen (PSA) response rates and radiographic responses [3]. Retrospective as well as early prospective data confirmed the favourable safety profile of RLT $[4,5]$.

Although up to $60 \%$ of the patients showed some degree of response to RLT, there are patients who do not benefit from this treatment $[4,5]$. The presence of visceral metastases, alkaline phosphatase (ALP) $\geq$ $220 \mathrm{U} / \mathrm{L}$ [5], high platelet count, a regular need for analgesics [6] or elevated lactate dehydrogenase [7] have been identified as negative predictors for the outcome, whereas ALP $<220 \mathrm{U} / \mathrm{L}$, a cumulative injected activity $\geq 18.8 \mathrm{GBq}$ [8], albumin $\geq 38.6 \mathrm{~g} / \mathrm{L}$, aspartate transaminase (AST) $\leq 24 \mathrm{U} / \mathrm{L}$, 
haemoglobin $\geq 10.4 \mathrm{~g} / \mathrm{dL}$, absence of liver metastases [9], and higher standardized uptake value (SUV) mean or max in positron emission tomography (PET) with PSMA ligands have been reported to be associated with favourable outcome [10]. However, the predictive value of these factors varies significantly between studies.

The aim of this study was first, to identify predictive factors for the outcome of RLT with [ ${ }^{177}$ Lu]Lu-PSMA considering laboratory parameters, medical history and imaging obtained before first RLT and second, to derive a predictive score which can simplify the selection of patients who are or are not likely to benefit from RLT.

\section{Materials And Methods Patients}

Between 06/2015 and 12/2018, 91 patients with mCRPC consecutively underwent RLT with [ $\left.{ }^{177} \mathrm{Lu}\right] \mathrm{Lu}$ PSMA in the department of nuclear medicine of our university hospital.

Inclusion criteria were: 1 ) at least two cycles of RLT with [ $\left.{ }^{177} \mathrm{Lu}\right] \mathrm{Lu}-\mathrm{PSMA}$, 2) $\left[{ }^{68} \mathrm{Ga}\right] \mathrm{Ga}-\mathrm{PSMA}$ positron emission tomography (PET) / computed tomography (CT) examination in the same department $\leq 8$ weeks prior to first cycle of RLT and 3) complete patient records including follow-up for at least 2 months after second cycle RLT. 45 patients were excluded for the following reasons: PSMA-PET prior to RLT in other facilities or non-PET imaging with PSMA-ligands $(n=43)$ and absence of follow-up after RLT $(n=2)$, Thus, the final study population included 46 patients.

Patients records were assessed for the medical history including age at the time point of RLT, time between first diagnosis and RLT, initial Gleason score, initial D'Amico risk classification [11], presence of distant metastases at the time of initial diagnosis, treatment before RLT and type of metastases at time of RLT. Baseline laboratory tests including liver, renal and hematologic function, electrolytes, PSA and testosterone levels were recorded. Imaging parameters from ${ }^{68} \mathrm{Ga}-\mathrm{PSMA} P E T / C T$ including maximum and mean standard uptake value (SUVmax and SUVmean) of the lesion with the highest tracer uptake and the visual tumor burden were also determined. Visual tumor burden was subdivided into three categories: low (number of focal lesions in PET/CT $<10$ ), medium (number of lesions in PET/CT $>10$ and $<30$ ) and high (disseminated metastatic spread).

\section{Treatment, imaging, laboratory and response assessment}

RLT with [ $\left.{ }^{177} \mathrm{Lu}\right] \mathrm{Lu}$-PSMA and imaging with $\left[{ }^{68} \mathrm{Ga}\right] \mathrm{Ga}-\mathrm{PSMA}$ PET/CT were performed according to consensus recommendation or as previously described $[12,13]$. Laboratory parameters for liver, renal and hematologic function, electrolytes, PSA and testosterone levels were collected at each RLT cycle. In line with the German multicenter study for RLT with ${ }^{177}$ Lu-PSMA [5], evaluation of the treatment response was based on the change in PSA levels between baseline at the first RLT cycle and two months after the second RLT cycle. Treatment response was defined according to Prostate Cancer Clinical Trials Working 
Group 2 and 3 as follows: partial response (PR): $>50 \%$ decline; progressive disease (PD): $\geq 25 \%$ increase; stable disease (SD): $\leq 50 \%$ decline and $<25 \%$ increase of PSA level $[14,15]$.

\section{Statistical Analysis}

Statistical analyses were performed using SPSS 25 (IBM Corporation, Armonk, NY, USA). To evaluate the association between single factors and PD under RLT, receiver operating characteristic (ROC) curve analysis was performed for each factor. For factors with an area under the curve (AUC) $>0.65$ or $<0.35$ and a p-value $<0.05$, the optimal cut off value was determined (Youden's index) for binarization, and Fisher's exact test for PD vs. SD/PR was performed. P-values for each factor were corrected for multiple comparisons using the Benjamini-Hochberg procedure [16]. A p-value $<0.05$ was considered significant. The combined predictive score included all single factors with equal weighting that had shown significant association with PD in Fisher's exact test.

\section{Results}

\section{Patient, Treatment and Response Data}

The median patient age was 68 years (range, 53-87). The median PSA value before RLT was $79 \mathrm{ng} / \mathrm{ml}$ (range, 4-3133). Patient characteristics including previous treatments and relevant baseline parameters are summarized in Table 1. 
Table 1

Patient characteristics.

\begin{tabular}{|c|c|}
\hline Characteristic & Number (\% or range) \\
\hline Total patient number & 46 \\
\hline Median age [years] & $68(50-87)$ \\
\hline Median baseline PSA [ng/mL] & $79(4-3133)$ \\
\hline Median Gleason score & $9(6-10)$ \\
\hline Initial D’Amico group: & $3(6)$ \\
\hline Low-risk & $4(9)$ \\
\hline Intermediate-risk & $39(85)$ \\
\hline \multicolumn{2}{|l|}{ High-risk } \\
\hline Median time since first diagnosis [months] & 60 months (10 to 140 ) \\
\hline Extent at first diagnosis & $30(65)$ \\
\hline Localized & $16(35)$ \\
\hline \multicolumn{2}{|l|}{ Distant metastasized } \\
\hline Previous therapy & $24(52)$ \\
\hline RPX & $26(57)$ \\
\hline Radiation therapy & $46(100)$ \\
\hline Androgen-deprivation therapy & $28(61)$ \\
\hline Abiraterone & $26(57)$ \\
\hline Enzalutamide & $29(63)$ \\
\hline Chemotherapy & $14(30)$ \\
\hline${ }^{223}$ Radium or / and radiotherapy (bone) & $8(17)$ \\
\hline \multicolumn{2}{|l|}{ Others (including e.g. immunotherapy) } \\
\hline Site of metastases at first RLT & $39(85)$ \\
\hline Lymph nodes & $44(96)$ \\
\hline Bone & $7(15)$ \\
\hline Liver & $14(30)$ \\
\hline \multicolumn{2}{|l|}{ Visceral including liver } \\
\hline Median baseline ALP [U/L] & $119(54-1188)$ \\
\hline
\end{tabular}




\begin{tabular}{|ll|}
\hline Characteristic & Number (\% or range) \\
\hline Median baseline SUVmax & $47(5-133)$ \\
\hline Median baseline SUVmean & $21(4-49)$ \\
\hline Visual tumor burden & $18(39)$ \\
Low & $10(22)$ \\
Median & $18(39)$ \\
High & \\
\hline
\end{tabular}

Overall, 117 treatment cycles were performed with a median activity of $6 \mathrm{GBq}$ (range, 2.9-6.2) [177 Lu]LuPSMA per cycle. 3 patients underwent four, 19 patients three and 24 patients underwent two RLT cycles.

After the 2nd RLT cycle, fourteen patients (30\%) demonstrated PR with median PSA decrease of 73\%, sixteen patients (35\%) showed SD with median PSA decrease of $17 \%$, and sixteen patients $(35 \%)$ developed PD with median PSA increase of $42 \%$. The median overall survival was 19.4 months (range, 5.0-42.5) from 1st RLT cycle.

\section{Predictors of Treatment Response}

According to the predefined selection criteria, among all the analysed parameters only PSA, ALP, SUVmax and SUVmean were evaluated further for their suitability as predictive factors. Results of the ROC analysis are summarized in Table 2. 
Table 2

ROC curve analysis for PD after RLT.

\begin{tabular}{|c|c|c|}
\hline Parameter & AUC (95\% confidence interval) & p-value \\
\hline Age & $0.59(0.43-0.76)$ & 0.299 \\
\hline Time since first diagnosis & $0.58(0.41-0.75)$ & 0.393 \\
\hline Gleason score & $0.41(0.23-0.59)$ & 0.299 \\
\hline D’Amico risk & $0.42(0.24-0.60)$ & 0.393 \\
\hline Extent at first diagnosis & $0.47(0.30-0.65)$ & 0.764 \\
\hline Abiraterone & $0.66(0.49-0.82)$ & 0.080 \\
\hline Enzalutamide & $0.52(0.34-0.69)$ & 0.854 \\
\hline Chemotherapy & $0.58(0.41-0.76)$ & 0.356 \\
\hline Lymph node metastases & $0.43(0.25-0.61)$ & 0.406 \\
\hline Bone metastases & $0.53(0.36-0.41)$ & 0.712 \\
\hline Liver metastases & $0.62(0.44-0.80)$ & 0.174 \\
\hline Visceral metastases (including liver) & $0.60(0.43-0.78)$ & 0.258 \\
\hline PSA & $0.84(0.69-0.99)$ & 0.003 \\
\hline Serum creatinine & $0.56(0.39-0.73)$ & 0.518 \\
\hline Aspartate transaminase & $0.63(0.45-0.80)$ & 0.159 \\
\hline Alanine transaminase & $0.53(0.33-0.73)$ & 0.747 \\
\hline ALP & $0.73(0.54-0.93)$ & 0.041 \\
\hline Gamma-glutamyltransferase & $0.67(0.46-0.89)$ & 0.130 \\
\hline Blood urea nitrogen & $0.45(0.28-0.63)$ & 0.612 \\
\hline Uric acid & $0.57(0.29-0.85)$ & 0.611 \\
\hline Leucocytes & $0.42(0.24-0.59)$ & 0.344 \\
\hline Erythrocytes & $0.41(0.23-0.59)$ & 0.310 \\
\hline Thrombocytes & $0.37(0.20-0.54)$ & 0.153 \\
\hline Hemoglobin & $0.42(0.24-0.59)$ & 0.356 \\
\hline SUVmax & $0.29(0.13-0.46)$ & 0.022 \\
\hline SUVmean & $0.30(0.14-0.47)$ & 0.029 \\
\hline
\end{tabular}




\begin{tabular}{|lcl|}
\hline Parameter & AUC (95\% confidence interval) & p-value \\
\hline Visual tumor burden & $0.47(0.29-0.65)$ & 0.747 \\
\hline Statistically significant results are printed in bold. & \\
\hline
\end{tabular}

Table 3 shows the results of the Fisher's exact test for the aforementioned parameters with their corresponding optimal cut-off values. All factors remained statistically significant after BenjaminiHochberg adjustment. SUVmean was not considered in the predictive score because it did not show superior predictive value compared to the SUVmax while demonstrating lower inter-rater reliability due to the non-standardized measurement. Thus, the predictive score included baseline PSA > $200 \mathrm{ng} / \mathrm{ml}, \mathrm{AP}>$ $135 \mathrm{U} / \mathrm{I}$, and SUVmax $<45$, equally weighted.

Table 3

Fisher's exact test for association of single variables with PD after RLT.

\begin{tabular}{|llll|}
\hline Parameter & Patients (SD + PR) & Patients (PD) & 2-sided p-value \\
\hline PSA & $5(11 \%)$ & $8(18 \%)$ & 0.036 \\
$>200 \mathrm{ng} / \mathrm{mL}$ & $25(54 \%)$ & $8(17 \%)$ & \\
$\leq 200 \mathrm{ng} / \mathrm{mL}$ & & & \\
ALP & $4(9 \%)$ & $10(22 \%)$ & 0.030 \\
$>135 \mathrm{ng} / \mathrm{mL}$ & $26(56 \%)$ & $6(13 \%)$ & \\
$\leq 135 \mathrm{ng} / \mathrm{mL}$ & & & \\
SUVmax & $10(22 \%)$ & $13(28 \%)$ & 0.005 \\
$<45$ & $20(43 \%)$ & $3(7 \%)$ & \\
$\geq 45$ & & & 0.013 \\
SUVmean & $4(9 \%)$ & $8(18 \%)$ & \\
$<13$ & $26(56 \%)$ & $8(17 \%)$ & \\
$\geq 13$ & & & \\
\hline
\end{tabular}

Among 46 patients, 36 showed $\leq 2$ risk factors and 10 showed 3 risk factors. Among the 36 patients with $\leq 2$ risk factors, 29 patients (81\%) had SD or PR compared to 7 patients (19\%) with PD. In 10 patients with 3 risk factors, 1 patient (10\%) showed SD or PR while 9 patients $(90 \%)$ developed PD (Fisher's exact test, $p=0.009$ ).

\section{Discussion}

To the best of our knowledge, this is the first simple clinical score to predict the outcome after the first two cycles of [ $\left.{ }^{177} \mathrm{Lu}\right]$ Lu-PSMA in patients with mCRPC. The score combines laboratory (PSA, ALP) and 
imaging (SUVmax) parameters and can identify patients likely to benefit from RLT and potential nonresponders to RLT.

MCRPC patients with higher PSA levels have a higher likelihood to develop PD under treatment than patients with lower PSA levels. Especially, a high baseline serum PSA level of $>200 \mathrm{ng} / \mathrm{ml}$ seems to be a significant predictor for unfavorable outcome. This is consistent with the results of former studies showing that serum high PSA levels correlate strongly with the risk of prostate cancer progression, not only in the initial stage of disease, but also in advanced disease [17].

In the present study, a high ALP of $>135 \mathrm{U} / \mathrm{I}$ was also defined as a significant predictive factor for unfavorable outcome after RLT. This is in line with previous studies showing a poor outcome after RLT with [ ${ }^{177}$ Lu]Lu-PSMA in patients with elevated $\operatorname{ALP}[5,8,18]$. Similar outcome has been also reported for patients treated with chemotherapy [19]. ALP is a marker for bone turnover [19], which has led to the hypothesis that a high ALP correlates with progressive bone metastases.

Low baseline SUVmax $(<45)$ in $\left[{ }^{68} \mathrm{Ga}\right] \mathrm{Ga}$-PSMA PET also suggested an unfavorable outcome after RLT in the current study. The SUVmax/mean denotes the maximum/mean uptake in a specified region of interest (e.g., a lesion) and is the most commonly used semiquantitative parameter in PET. This may be explained by the requirement for a targeted therapy of a minimum level of PSMA expression on the cell surface to achieve adequate lutetium internalization into the cell. The SUVmax of a radiotracer has a significant role in the diagnosis and treatment selection for different types of tumors, e.g. lymphoma [20], head and neck squamous cell carcinoma [21], pancreatic tumor [22], neuroendocrine tumors [23] or even prostate cancer [24]. A recent pilot study on 14 prostate cancer patients showed that patients with a PSA response $\geq 50 \%$ after treatment with [ ${ }^{177} \mathrm{Lu}$ ]-PSMA had a significantly higher baseline SUVmax in a pretherapeutic scan with $\left[{ }^{68} \mathrm{Ga}\right.$ ]-PSMA-PET (44 vs. 17) [10]. This study also reported significantly higher SUVmean in patients with PSA response $\geq 50 \%$ (10 vs. 6) [9]. Likewise, SUVmean in [ ${ }^{68} \mathrm{Ga}$ ]Ga-PSMA-PET was a significant predictive factor of response in the present analysis. However, it was not included in the combined predictive score because of its high interrater variability and limited reproducibility $[25,26]$.

Not all potential imaging parameters were examined in our study, e.g. textural heterogeneity was recently postulated as a predictive factor in [ ${ }^{177}$ Lu]Lu-PSMA treatment [27]. Our goal was however, to derive a predictive score that is simple and intuitive for clinical use. Hence, only parameters which are determined routinely and quickly before treatment were analyzed and included.

Clear limitations of this study are the small sample size and the retrospective design. Our results are hypothesis generating and need to be confirmed in further studies.

\section{Conclusion}

In this study, a score for easy clinical use to predict the treatment response to [ $\left.{ }^{177} \mathrm{Lu}\right] \mathrm{Lu}$-PSMA based on pretreatment PSA, ALP, and SUVmax of the "hottest lesion" in $\left[{ }^{68} \mathrm{Ga}\right] \mathrm{Ga}$-PSMA-PET is presented, so that 
response rates of RLT can be increased and the rate of unnecessary adverse events can be reduced. This is a hypothesis generating study, further trials in independent cohorts are needed to test and validate this predictive score.

\section{Abbreviations}

ALP: alkaline phosphatase, AUC: area under the curve, CT: computed tomography, mCRPC: metastatic castrations-resistant prostate cancer, PD: progressive disease, PET: positron emission tomography, PR: partial remission, PSA: prostate specific antigen, PSMA: prostate specific membrane antigen, RLT radioligand therapy, ROC: receiver operating characteristics, SD: stable disease, SUVmax: maximum standardized uptake value, SUVmean: mean standardized uptake value

\section{Declarations}

\section{Ethical approval and consent to participate}

All procedures performed in studies involving human participants were in accordance with the ethical standards of the institutional and/or national research committee and with the 1964 Helsinki declaration and its later amendments or comparable ethical standards. This single-institution retrospective study was approved by the institutional ethics review board (EA2/177/17). All patients signed a written informed consent form for the study.

\section{Consent for publication}

Consent for publication was obtained from all individual participants included in the study.

\section{Availability of data and materials}

The datasets used and/or analysed during the current study are available from the corresponding author on reasonable request.

\section{Competing interests}

The authors declare that they have no competing interests.

\section{Funding}

None.

\section{Authors' contributions}

$\mathrm{KH}, \mathrm{IS}, \mathrm{WB}$ and HA were responsible for the study concept and design. $\mathrm{KH}, \mathrm{HA}, \mathrm{MDS}$ and BE carried out the data acquisition and were responsible for the organisational procedures between the Departments of Urology and Nuclear Medicine. JMMR supervised the statistical analyses. KH + HA prepared the 
manuscript. KH wrote the manuscript. KH, IS, JMMR, RLL, MDS, BE, PR, WB, HA edited and revised the manuscript. All authors read and approved the final manuscript.

\section{Acknowledgements}

Not applicable.

\section{References}

1. Ferlay J, Colombet M, Soerjomataram I, Dyba T, Randi G, Bettio M, et al. Cancer incidence and mortality patterns in Europe: Estimates for 40 countries and 25 major cancers in 2018. Eur J Cancer. 2018;103:356-87. doi:10.1016/j.ejca.2018.07.005.

2. Crawford ED, Higano CS, Shore ND, Hussain M, Petrylak DP. Treating Patients with Metastatic Castration Resistant Prostate Cancer: A Comprehensive Review of Available Therapies. J Urol. 2015;194:1537-47. doi:10.1016/j.juro.2015.06.106.

3. Yadav MP, Ballal S, Bal C, Sahoo RK, Damle NA, Tripathi M, et al. Efficacy and Safety of 177Lu-PSMA617 Radioligand Therapy in Metastatic Castration-Resistant Prostate Cancer Patients. Clin Nucl Med. 2020;45:19-31. doi:10.1097/RLU.0000000000002833.

4. Hofman MS, Violet J, Hicks RJ, Ferdinandus J, Thang SP, Akhurst T, et al. [(177)Lu]-PSMA-617 radionuclide treatment in patients with metastatic castration-resistant prostate cancer (LuPSMA trial): a single-centre, single-arm, phase 2 study. Lancet Oncol. 2018;19:825-33. doi:10.1016/S14702045(18)30198-0.

5. Rahbar K, Ahmadzadehfar H, Kratochwil C, Haberkorn U, Schafers M, Essler M, et al. German Multicenter Study Investigating 177Lu-PSMA-617 Radioligand Therapy in Advanced Prostate Cancer Patients. J Nucl Med. 2017;58:85-90. doi:10.2967/jnumed.116.183194.

6. Ferdinandus J, Eppard E, Gaertner FC, Kurpig S, Fimmers R, Yordanova A, et al. Predictors of Response to Radioligand Therapy of Metastatic Castrate-Resistant Prostate Cancer with 177LuPSMA-617. J Nucl Med. 2017;58:312-9. doi:10.2967/jnumed.116.178228.

7. Heck MM, Tauber R, Schwaiger S, Retz M, D'Alessandria C, Maurer T, et al. Treatment Outcome, Toxicity, and Predictive Factors for Radioligand Therapy with (177)Lu-PSMA-I\&T in Metastatic Castration-resistant Prostate Cancer. Eur Urol. 2018;75:920-6. doi:10.1016/j.eururo.2018.11.016.

8. Rahbar K, Boegemann M, Yordanova A, Eveslage M, Schafers M, Essler M, et al. PSMA targeted radioligandtherapy in metastatic castration resistant prostate cancer after chemotherapy, abiraterone and/or enzalutamide. A retrospective analysis of overall survival. Eur J Nucl Med Mol Imaging. 2018;45:12-9. doi:10.1007/s00259-017-3848-4.

9. Ahmadzadehfar H, Schlolaut S, Fimmers R, Yordanova A, Hirzebruch S, Schlenkhoff C, et al. Predictors of overall survival in metastatic castration-resistant prostate cancer patients receiving [(177)Lu]Lu-PSMA-617 radioligand therapy. Oncotarget. 2017;8:103108-16.

doi:10.18632/oncotarget.21600. 
10. Emmett L, Crumbaker M, Ho B, Willowson K, Eu P, Ratnayake L, et al. Results of a Prospective Phase 2 Pilot Trial of (177)Lu-PSMA-617 Therapy for Metastatic Castration-Resistant Prostate Cancer Including Imaging Predictors of Treatment Response and Patterns of Progression. Clin Genitourin Cancer. 2019;17:15-22. doi:10.1016/j.clgc.2018.09.014.

11. D'Amico AV, Whittington R, Malkowicz SB, Schultz D, Blank K, Broderick GA, et al. Biochemical outcome after radical prostatectomy, external beam radiation therapy, or interstitial radiation therapy for clinically localized prostate cancer. JAMA. 1998;280:969-74. doi:10.1001/jama.280.11.969.

12. Fendler WP, Kratochwil C, Ahmadzadehfar H, Rahbar K, Baum RP, Schmidt M, et al. [177Lu-PSMA-617 therapy, dosimetry and follow-up in patients with metastatic castration-resistant prostate cancer]. Nuklearmedizin. 2016;55:123-8.

13. Rogasch JM, Cash H, Zschaeck S, Elezkurtaj S, Brenner W, Hamm B, et al. Ga-68-PSMA PET/CT in treatment-naive patients with prostate cancer: Which clinical parameters and risk stratification systems best predict PSMA-positive metastases? Prostate. 2018;78:1103-10. doi:10.1002/pros.23685.

14. Scher HI, Halabi S, Tannock I, Morris M, Sternberg CN, Carducci MA, et al. Design and end points of clinical trials for patients with progressive prostate cancer and castrate levels of testosterone: recommendations of the Prostate Cancer Clinical Trials Working Group. J Clin Oncol. 2008;26:114859. doi:10.1200/JCO.2007.12.4487.

15. Scher HI, Morris MJ, Stadler WM, Higano C, Basch E, Fizazi K, et al. Trial Design and Objectives for Castration-Resistant Prostate Cancer: Updated Recommendations From the Prostate Cancer Clinical Trials Working Group 3. J Clin Oncol. 2016;34:1402-18. doi:10.1200/JC0.2015.64.2702.

16. Victor A, Elsasser A, Hommel G, Blettner M. Judging a plethora of p-values: how to contend with the problem of multiple testing-part 10 of a series on evaluation of scientific publications. Dtsch Arztebl Int. 2010;107:50-6. doi:10.3238/arztebl.2010.0050.

17. Stephan C, Ralla B, Jung K. Prostate-specific antigen and other serum and urine markers in prostate cancer. Biochim Biophys Acta. 2014;1846:99-112. doi:10.1016/j.bbcan.2014.04.001.

18. Brauer A, Grubert LS, Roll W, Schrader AJ, Schafers M, Bogemann M, et al. (177)Lu-PSMA-617 radioligand therapy and outcome in patients with metastasized castration-resistant prostate cancer. Eur J Nucl Med Mol Imaging. 2017;44:1663-70. doi:10.1007/s00259-017-3751-Z.

19. Wajsman Z, Chu TM, Bross D, Saroff J, Murphy GP, Johnson DE, et al. Clinical significance of serum alkaline phosphatase isoenzyme levels in advanced prostatic carcinoma. J Urol. 1978;119:244-6. doi:10.1016/s0022-5347(17)57446-7.

20. Cheson BD, Fisher RI, Barrington SF, Cavalli F, Schwartz LH, Zucca E, et al. Recommendations for initial evaluation, staging, and response assessment of Hodgkin and non-Hodgkin lymphoma: the Lugano classification. J Clin Oncol. 2014;32:3059-68. doi:10.1200/JC0.2013.54.8800.

21. Cacicedo J, Fernandez I, Del Hoyo O, Navarro A, Gomez-Iturriaga A, Pijoan JI, et al. Prognostic value of maximum standardized uptake value measured by pretreatment 18F-FDG PET/CT in locally 
advanced head and neck squamous cell carcinoma. Clin Transl Oncol. 2017;19:1337-49. doi:10.1007/s12094-017-1674-6.

22. Pergolini I, Crippa S, Salgarello M, Belfiori G, Partelli S, Ruffo G, et al. SUVmax after (18)fluorodeoxyglucose positron emission tomography/computed tomography: A tool to define treatment strategies in pancreatic cancer. Dig Liver Dis. 2018;50:84-90. doi:10.1016/j.dld.2017.09.122.

23. Kratochwil C, Stefanova M, Mavriopoulou E, Holland-Letz T, Dimitrakopoulou-Strauss A, AfsharOromieh A, et al. SUV of [68Ga]DOTATOC-PET/CT Predicts Response Probability of PRRT in Neuroendocrine Tumors. Mol Imaging Biol. 2015;17:313-8. doi:10.1007/s11307-014-0795-3.

24. Basha MAA, Hamed MAG, Hussein O, El-Diasty T, Abdelkhalek YI, Hussein YO, et al. (68)Ga-PSMA-11 $\mathrm{PET} / \mathrm{CT}$ in newly diagnosed prostate cancer: diagnostic sensitivity and interobserver agreement. Abdom Radiol (NY). 2019;44:2545-56. doi:10.1007/s00261-019-02006-2.

25. Huang YE, Chen CF, Huang YJ, Konda SD, Appelbaum DE, Pu Y. Interobserver variability among measurements of the maximum and mean standardized uptake values on (18)F-FDG PET/CT and measurements of tumor size on diagnostic CT in patients with pulmonary tumors. Acta Radiol. 2010;51:782-8. doi:10.3109/02841851.2010.497772.

26. Jauw YWS, Bensch F, Brouwers AH, Hoekstra OS, Zijlstra JM, Pieplenbosch S, et al. Interobserver reproducibility of tumor uptake quantification with (89)Zr-immuno-PET: a multicenter analysis. Eur J Nucl Med Mol Imaging. 2019;46:1840-9. doi:10.1007/s00259-019-04377-6.

27. Khurshid Z, Ahmadzadehfar H, Gaertner FC, Papp L, Zsoter N, Essler M, et al. Role of textural heterogeneity parameters in patient selection for 177Lu-PSMA therapy via response prediction. Oncotarget. 2018;9:33312-21. doi:10.18632/oncotarget.26051. 